\title{
Abundance of gut Prevotella at baseline and metabolic response to barley prebiotics
}

\author{
Jonna Sandberg ${ }^{1,2}\left[\right.$ Petia Kovatcheva-Datchary ${ }^{3} \cdot$ Inger Björck $^{2} \cdot$ Fredrik Bäckhed $^{3,4} \cdot$ Anne Nilsson ${ }^{1,2}$
}

Received: 21 February 2018 / Accepted: 17 July 2018 / Published online: 25 July 2018

(c) The Author(s) 2018

\begin{abstract}
Purpose We previously showed that short-term intervention with barley kernel bread (BKB) improved glucose tolerance. However, glucose tolerance was not improved in a subset of individuals (non-responders) who were characterized by a low Prevotella/Bacteroides ratio. The purpose of the present study was to investigate if the baseline Prevotella/Bacteroides ratio can be used to stratify metabolic responders and non-responders to barley dietary fiber (DF).

Methods Fecal samples were collected from 99 healthy humans with $\mathrm{BMI}<28 \mathrm{~kg} / \mathrm{m}^{2}$ between 50 and 70 years old. The abundance of fecal Prevotella and Bacteroides was quantified with 16S rRNA quantitative PCR. 33 subjects were grouped in three groups: subjects with highest Prevotella/Bacteroides ratios, "HP", $n=12$; subjects with lowest Prevotella/Bacteroides ratios, "LP", $n=13$; and subjects with high abundance of both measured bacteria, HPB, $n=8$. A 3-day randomized crossover intervention with BKB and white wheat bread (control) was performed. Cardiometabolic test variables were analyzed the next day following a standardized breakfast.

Results The BKB intervention lowered the blood glucose responses to the breakfast independently of Prevotella/Bacteroides ratios $(P<0.01)$. However, independently of intervention, the HP group displayed an overall lower insulin response and lower IL-6 concentrations compared with the LP group $(P<0.05)$. Furthermore, the groups HP and HPB showed lower hunger sensations compared to the LP group $(P<0.05)$.

Conclusions Here we show that the abundance of gut Prevotella and Bacteroides at baseline did not stratify metabolic responders and non-responders to barley DF intervention. However, our results indicate the importance of gut microbiota in host metabolic regulation, further suggesting that higher Prevotella/Bacteroides ratio may be favorable.
\end{abstract}

ClinicalTrials.gov ID NCT02427555

Keywords Prevotella $\cdot$ Bacteroides $\cdot$ Barley $\cdot$ Prevention $\cdot$ Stratification $\cdot$ Glucose regulation

\section{Introduction}

Electronic supplementary material The online version of this article (https://doi.org/10.1007/s00394-018-1788-9) contains supplementary material, which is available to authorized users.

Jonna Sandberg

jonna.sandberg@food.lth.se

Petia Kovatcheva-Datchary

petia.kovatcheva@wlab.gu.se

Inger Björck

inger@innovafood.se

Fredrik Bäckhed

fredrik.backhed@wlab.gu.se

Anne Nilsson

anne.nilsson@food.lth.se
Lifestyle habits play a pivotal role for the development of obesity, type 2 diabetes (T2D) and cardiovascular disease (CVD) [1]. Diet is probably the most significant lifestyle

1 Department of Food Technology, Engineering and Nutrition, Lund University, Lund, Sweden

2 Food for Health Science Centre, Lund University, Lund, Sweden

3 Wallenberg Laboratory, Department of Molecular and Clinical Medicine, University of Gothenburg, Gothenburg, Sweden

4 Faculty of Health and Medical Sciences, Novo Nordisk Foundation Center for Basic Metabolic Research, University of Copenhagen, Copenhagen, Denmark 
factor for the etiology of metabolic diseases, making the dietary composition an essential target in a strategy aiming at prevention [1]. Recent studies have demonstrated that saturated fats and animal protein, which are typically high in a Western diet, are associated with metabolic disorders such as obesity, reduced insulin sensitivity, T2D, and CVD [2], and also altered microbiota, e.g., reduced diversity [3]. In contrast, inclusion of whole grain foods in the diet has been found advantageous by lowering the risk of weight gain, T2D, and CVD [4]. Accumulating evidence from both epidemiological studies [5] and dietary interventions [6-9] suggest preventive potential of dietary fiber (DF) on cardiometabolic risk. The beneficial effects of DF are largely suggested to emanate from mechanisms related to microbial metabolism of the DF [6-10]. We and others have shown that barley kernel-based evening meals improve glucose tolerance following a subsequent standardized breakfast $[6$, $7,10]$. The underlying mechanisms were suggested to emanate from colonic fermentation of the DF present in barley. Recent studies have observed a high variability in the effects on glucose tolerance to food between subjects although the meals or more specific food items were identical [11, 12]. Thus, the importance of personalized nutrition is highlighted and it has been suggested that the glucose response can be predicted by collecting data regarding personal features and/ or gut microbiota composition $[11,12]$. In agreement, we previously reported high variability in glucose response in test subjects following barley kernel-based evening meal [10]. Interestingly, we found that the microbiota differed between the subjects acquiring improved glucose tolerance after intake of barley (responder subjects) compared to subjects that showed no or minor effects on glucose tolerance (non-responder subjects). In responder subjects, intake of barley was associated with increased abundance of gut Prevotella and increased ratio of Prevotella/Bacteroides. In contrast, in non-responders, the abundance of Prevotella or ratio Prevotella/Bacteroides was not affected after barley kernel-based products. In addition, we observed tendencies towards an increased ratio of Prevotella/Bacteroides in the responder subjects compared with the non-responder subjects already at baseline, i.e., prior to the barley intervention [10]. The purpose of this study was to investigate if the ratio Prevotella/Bacteroides at baseline can be used to identify and stratify metabolic responders from non-responders to barley DF in a population.

\section{Materials and methods}

\section{Test subjects}

Recruitments of subjects were conducted through advertisements in local newspapers during May and June 2015.
The screening was conducted at the Food for Health Science Centre, Lund University. The inclusion criteria were as follows: men and women aged 50-70 years with normal to slightly increased body mass index $\left(\mathrm{BMI}<28 \mathrm{~kg} / \mathrm{m}^{2}\right)$, normal blood glucose concentration as determined with a random blood glucose test $<8.7 \mathrm{mmol} / \mathrm{l}$, overall healthy and with unknown metabolic disorders or food allergies. Subjects with anti-hypertensive medications (3 subjects) and prescription-free painkillers without anti-inflammatory action were allowed to participate. In addition, no antibiotics or probiotics should have been consumed within 3 weeks prior to fecal donation, during the selection process and throughout the following dietary intervention. In total, 99 apparently healthy volunteers (as judged from the inclusion criteria), 25 men and 74 women, aged 64.1 (SD 5.6) years, with a BMI of $24.3 \mathrm{~kg} / \mathrm{m}^{2}\left(\mathrm{SD} 3.4 \mathrm{~kg} / \mathrm{m}^{2}\right)$ and with a random blood glucose concentration of $5.5 \mathrm{mmol} / \mathrm{l}(\mathrm{SD} 0.8 \mathrm{mmol} / \mathrm{l}$ ), donated stool samples at baseline, i.e., prior to start of the dietary intervention.

The study was conducted in accordance to the Declaration of Helsinki, and all procedures involving human subjects were approved by the Regional Ethical Review Board in Lund, Sweden (Reference 2015/58). Written informed consent was obtained from all the subjects. The study was registered at ClinicalTrials.gov (NCT02427555).

\section{Genomic DNA purification and 16S rRNA quantitative PCR analysis}

Fecal samples were collected at baseline from all 99 subjects. The sample was immediately frozen $\left(-20{ }^{\circ} \mathrm{C}\right)$ and handed over to the experimental department within $24 \mathrm{~h}$ for continued storage at $-80^{\circ} \mathrm{C}$ until analysis. Genomic DNA was purified from the feces of 99 subjects, using repeated bead beating [13]. 16S rRNA quantitative PCR was performed with a CFX96 Real-Time System (Bio-Rad Laboratories). Samples were analyzed in a $25-\mu$ l reaction mix consisting of $12.5 \mu \mathrm{l} 1 \mathrm{xSYBR}$ Green Master Mix buffer (Thermo Scientific, Waltham, Massachusetts, USA), $0.2 \mu \mathrm{M}$ of each primer and $5 \mu \mathrm{l}$ of template DNA, water or genomic DNA. All reactions were performed in duplicate in one run. qPCR conditions were as reported previously: for Bacteroides and universal [14]; for Prevotella [15]; Standard curves of $16 \mathrm{~S}$ rRNA PCR product of $B$. thetaiotaomicron, P. copri or Escherichia coli (for universal 16S rRNA qPCR) were created using serial tenfold dilution of the purified PCR product.

\section{Selection of individuals for the short-term dietary intervention}

The selection of the individuals for the intervention study with barley kernel bread (BKB) and white wheat bread 
(WWB) was based on their baseline microbiota composition. Main criteria for the subgrouping were the abundance (high 'H' or low 'L') of Prevotella and Bacteroides and the ratio of Prevotella/Bacteroides in their baseline gut microbiota. Individuals with highest levels of Prevotella and low levels of Bacteroides and ratio of Prevotella/Bacteroides more than 1.0 were identified as high Prevotella (HP) subjects. Individuals with low levels of Prevotella and high levels of Bacteroides and the ratio of Prevotella/Bacteroides equal to 0 were identified as low Prevotella (LP) subjects. Out of the total cohort of 99 subjects, we identified 15 HP subjects. Based on this number, we selected 15 LP subjects with the lowest Prevotella levels and the highest Bacteroides levels. Some of the individuals $(n=10)$ had high levels of Prevotella and high levels of Bacteroides and were identified as high Prevotella and Bacteroides (HPB) subjects and also included in the intervention study.

An additional inclusion criterion for participation in the short-term intervention study was that the fasting level of plasma glucose should be below $6.1 \mathrm{mmol} / \mathrm{l}$, i.e., below the level of impaired fasting glucose $(6.1-6.9 \mathrm{mmol} / \mathrm{l})$ according to the World Health Organization definition [16]. Six participants were excluded due to fasting plasma glucose $>6.4 \mathrm{mmol} / \mathrm{l}$ (LP, $n=2$; HP, $n=1$ and HPB, $n=1$ ) or a BMI $>30 \mathrm{~kg} / \mathrm{m}^{2}$ (HP, $n=1$ and HPB, $\left.n=1\right)$. Due to the exclusions, the statistical evaluations were based on 12 subjects in the HP group, 13 subjects in the LP group and 8 subjects in the group HPB.

\section{Test (BKB)—reference (WWB) products}

The test product $\mathrm{BKB}$ consisted of a barley kernel-based bread containing $85 \%$ barley kernels (Finax) and 15\% white wheat flour, presented as \% cereal dry matter (dm). The reference product WWB consisted of a white wheatbased bread and was based on $100 \%(\mathrm{dm})$ white wheat flour
(Kungsörnen AB). The WWB was also included in the standardized breakfast. The BKB and WWB were characterized with respect to total starch [17], resistant starch (RS) [18], and insoluble and soluble non-starch polysaccharides (NSP) [19]. Further details regarding characterization using these methods are provided elsewhere [6]. The starch and NSP compositions are presented in Table 1.

The ingredients and baking procedure of $\mathrm{BKB}$ and WWB are in accordance with previously used procedures [6], except that the barley kernels were boiled for $20 \mathrm{~min}$ instead of $12 \mathrm{~min}$ to obtain adequate cooked structure. After baking and cooling, the WWB was sliced into portion sizes and wrapped in aluminum foil, placed in plastic bags and stored in a freezer $\left(-20{ }^{\circ} \mathrm{C}\right)$. The $\mathrm{BKB}$ was placed in a plastic bag at room temperature overnight to obtain a firmer structure and facilitating the slicing into portions. The BKB then followed the procedure similar to the WWB. Before freezing, the crust was removed from the WWB included in the standardized breakfast. The subjects were instructed to take a daily portion of bread from the freezer on the day prior to consumption and to thaw it at ambient temperature, still wrapped in aluminum foil and maintained in the plastic bag. Study design and intervention protocol.

\section{Study design and intervention protocol}

The intervention study was conducted at the Food for Health Science Centre, Lund University, and was completed in May 2016. The study design was crossover, with 3-day dietary intervention periods with $\mathrm{BKB}$ and WWB reference, respectively, performed in a randomized order separated with a minimum of a 2-week wash-out period in-between intervention periods. During the wash-out period, the subjects returned to their normal eating habits without diet restrictions or intake of test products. The daily quantity ingested of the test $(\mathrm{BKB})$ and reference $(\mathrm{WWB})$ products during
Table 1 Characterization of test and reference product with respect to starch (total, available and resistant) and NSP

\begin{tabular}{|c|c|c|c|c|c|c|c|}
\hline \multicolumn{5}{|l|}{ Starch } & \multicolumn{2}{|l|}{ NSP } & \multirow[t]{2}{*}{ Total DF } \\
\hline Products & Daily amounts & Total & Available $^{\mathrm{a}}$ & $\mathrm{RS}$ & Insoluble & Soluble & \\
\hline \multicolumn{8}{|c|}{$\%$ dry matter } \\
\hline $\mathrm{BKB}$ & - & 65.5 & 57.2 & 8.4 & 8.4 & 4.0 & 20.8 \\
\hline WWB & - & 76.1 & 74.1 & 2.0 & 3.9 & 2.0 & 7.9 \\
\hline \multicolumn{8}{|l|}{ g/day } \\
\hline BKB & 384 & 115 & 100 & 14.7 & 14.7 & 7.0 & 36.4 \\
\hline WWB & 259 & 103 & 100 & 2.7 & 5.3 & 2.7 & 10.7 \\
\hline
\end{tabular}

Data are presented as means

$B K B$ barley kernel bread, $R S$ resistant starch, $N S P$ non-starch polysaccharides, $D F$ dietary fiber, $W W B$ white wheat bread

${ }^{a}$ Available starch was obtained by calculating the difference between total starch and RS. Values of total and available starch are based on means of 2 replications, RS means of 6 replications, DF means of 3 replications 
the 3-day study periods was equivalent to $100 \mathrm{~g}$ potentially available starch per day (Table 1). The daily amount of product was divided into three portions, and the subjects were instructed to distribute the daily intake of BKB or WWB over the day to suit their diet habits during day 1 and 2. On day 3 , half of the daily intake (i.e., $50 \mathrm{~g}$ available starch) was distributed equally between meals consumed in the morning and in the afternoon, and the other half (i.e., $50 \mathrm{~g}$ available starch) was consumed in the evening at 2100 hours.

The subjects were encouraged to standardize their meal pattern and to avoid alcohol and foods rich in DF during the 3-day intervention periods, and to avoid excessive physical activity on day 3. To facilitate the standardization of meal patterns and to monitor diet intake, the subjects were instructed to provide a meal record during the 3-day intervention period. After intake of the last portion of the BKB or WWB evening meal on day 3 , the subjects were fasting until the next morning when the standardized breakfast was provided at the research unit. The subjects arrived at the research unit at $07.30 \mathrm{am}$, and an intravenous cannula (BD Venflon; Becton Dickinson) was inserted into an antecubital vein for blood sampling. A fasting blood samples were obtained, and subjective appetite variables and breath hydrogen $\left(\mathrm{H}_{2}\right)$ determined, prior to the standardized breakfast provided at approximately $08.00 \mathrm{~h}$. The standardized breakfast corresponded to $50 \mathrm{~g}$ available carbohydrates and consisted of $129.6 \mathrm{~g} \mathrm{WWB}$, and was ingested together with $2.5 \mathrm{dl}$ tap water. The subjects were instructed to ingest the breakfast within $12 \mathrm{~min}$. During the following experimental time period, the subjects were instructed to maintain low physical activity.

\section{Collection and analysis of physiological variables}

Venous blood samples were collected to analyze the serum (s-) insulin and s-free fatty acids (FFA) and the plasma (p-) glucagon-like peptide-1 (GLP-1), p-GLP-2, p-peptide YY (PYY), p-interleukin-6 (IL-6) and p-c-reactive protein (CRP). Capillary blood samples were taken via finger prick test to determine whole blood glucose concentrations, presenting the results in plasma (HemoCue ${ }^{\circledR} \mathrm{B}$-glucose; HemoCue AB). Indication of colonic fermentation activity was measured as breath hydrogen $\left(\mathrm{H}_{2}\right)$ excretion using a Gastro+ (Bedfont EC60 Gastrolyzer; Bedfont). Measurements of subjective appetite sensations (satiety, hunger and desire to eat) were determined using a 100-mm visual analog scale.

Glucose, $\mathrm{H}_{2}$ and appetite sensations were obtained at fasting and at 15, 30, 45, 60, 90, 120, 150 and 180 min after the standardized breakfast. Insulin was analyzed at the same time points, excluding the $180 \mathrm{~min}$ time point. GLP-1 was analyzed at fasting and at 30, 60, 90, 120 and $180 \mathrm{~min}$. GLP2, PYY and IL-6 were analyzed at fasting and at 60, 120 and
$180 \mathrm{~min}$. FFA was determined at fasting and at $180 \mathrm{~min}$, and CRP was analyzed at fasting. The plasma tubes were kept on ice ( $\max 30 \mathrm{~min}$ ) and serum tubes at room temperature ( $\max$ $1 \mathrm{~h}$ room temperature), after blood collection, then centrifuged and immediately frozen after separation and stored in a freezer until analysis $\left(-40^{\circ} \mathrm{C}\right.$, except samples for GLP-1 which were kept in $\left.-80{ }^{\circ} \mathrm{C}\right)$. Assigned blood-collecting tubes for analysis of p-GLP-1, p-GLP-2 and p-PYY were prepared with a dipeptidyl peptidase-4 (DPPIV) inhibitor (10 $\mu \mathrm{l} / \mathrm{ml}$ blood) (Millipore). Tubes prepared with DPPIV inhibitor were kept cold until use, for a maximum of $24 \mathrm{~h}$.

Enzyme immunoassays were used for measuring levels of s-insulin (Mercodia Insulin ELISA, Mercodia AB, Uppsala, Sweden), p-CRP (CRP ELISA Kit, Immundiagnostik AG, Bensheim, Germany), p-IL-6 (Quantikine ${ }^{\circledR}$ HS ELISA, Human IL-6 High Sensitivity HS600B, R\&D Systems, Abingdon, UK), p-GLP-1 (GLP-1 (Active 7-36) ELISA 43-GP1HU-E01, ALPCO Diagnostics, Salem, NH), p-GLP-2 (Human GLP-2 EIA YK141, Yanaihara Institute Inc. Shizuoka, Japan) and p-PYY [both PYY (3-36) and PYY (1-36)] (Human PYY EIA YK080, Yanaihara Institute Inc. Shizuoka, Japan). Serum FFA levels were determined using an enzymatic colorimetric method (NEFA C, ACSACOD method; Wako Chemicals GmbH).

\section{Calculations and statistical methods}

The statistical evaluations were performed with respect to investigations of effects of treatment (BKB and WWB) and baseline gut microbiota composition (subgroups: HP, LP and HPB), respectively, and interactions thereof, on the test variables included. The effects of treatment and baseline gut microbiota composition, respectively, as well as [Meal * Microbiota] interactions, on physiological markers were analyzed with repeated measures ANOVA (general linear model) applying a $2 \times 3$ factorial design including two evening meals (BKB and WWB), i.e., 'Meal', and three baseline gut microbiota compositions (HP, LP and HPB), i.e., 'Microbiota', as independent main variables. The ANOVA was then followed by the post hoc analysis: Tukey's pairwise multiple comparison method (MINITAB Statistical Software). In addition, a second approach of the factorial design was performed to further elucidate the differences in effects on the physiological markers depending on highor low abundance of Prevotella and Bacteroides, excluding the HPB group. Thus, the 'Microbiota' variable in the $2 \times 2$ factorial design included only the two "extreme" groups with respect to ratios of Prevotella/Bacteroides at baseline, i.e., the HP and LP subgroups. Furthermore, the metabolic responses depending on treatment were also evaluated separately in each microbiota subgroup using one-way ANOVA analysis (MINITAB Statistical Software). 
Participants acted as their own control in the statistical evaluation. In the case of unevenly distributed residuals (tested using Anderson-Darling normality test and considered unevenly distributed if $P<0.05$ ), transformation using Box Cox was performed on the data prior to the ANOVA analysis. Order of consumption of test and reference product was randomized using the Random function in Microsoft Excel 2013 (Washington, USA). In case a value from a subject was missing for the test or reference product, the subject was excluded from that specific calculation. Data are expressed as means \pm SEM and values of $P<0.05$ are considered significant.

The statistical evaluations of glucose and insulin areas were based on both definite values and incremental changes from baseline to postprandial phase at the standardized breakfast consumed at day 4 (the day after the last portion of test product). Calculations of remaining test variables were performed using actual values. The trapezoid model was used to calculate the postprandial area and incremental area under the curve (AUC and iAUC, respectively) for each subject and test product. Graphs and calculations of areas were performed using Graph Prism (version 6; GraphPad Software). Postprandial mean values are reported instead of AUC and iAUC for test variables if the concentrations scarcely changed during the experimental day. Calculations of insulin resistance were made using a homoeostatic model assessment (HOMA-IR) formula [fasting glucose $(\mathrm{mmol} / \mathrm{l}) \times$ fasting insulin $(\mathrm{mU} / \mathrm{l}) / 22.5]$ [20]. Insulin sensitivity was assessed using a modified composite insulin sensitivity ( ISI $_{\text {composite }}$ ), also known as Matsuda index, and involved fasting and postprandial measures of b-glucose and

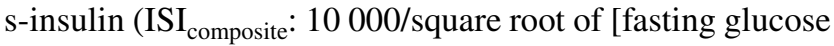
$(\mathrm{mg} / \mathrm{dl}) \times$ fasting insulin $(\mu \mathrm{U} / \mathrm{ml}) \times$ mean glucose concentrations $0-120 \mathrm{~min}(\mathrm{mg} \mathrm{min} / \mathrm{dl}) \times$ mean insulin concentrations 0-120 $\mathrm{min}(\mu \mathrm{U} \mathrm{min} / \mathrm{ml})]$ ) [21]. Modification of Matsuda index refers to that postprandial concentration of b-glucose and s-insulin was obtained after a WWB breakfast consisting of $50 \mathrm{~g}$ rapidly available starch instead of $75 \mathrm{~g}$ glucose.

\section{Results}

\section{Baseline abundance of gut bacteria and subject selection}

To investigate if the baseline gut microbiota composition, in particular the abundance of Prevotella and the ratio Prevotella/Bacteroides, can identify individuals that will respond to short-term barley prebiotic intervention with improved glucose metabolism, we first quantified the abundance of Prevotella and Bacteroides in feces of 99 subjects. From this cohort, we identified 15 subjects with highest abundance of Prevotella and lowest abundance of
Bacteroides (group HP). Based on this, we selected 15 subjects with the lowest abundance of Prevotella and highest abundance of Bacteroides that formed the LP group, and 10 subjects with high abundance of both bacteria (group HPB) to be included in the short-term barley intervention. Of the selected subjects, 12 HP subjects, 13 LP subjects and 8 HPB subjects (Table 2) completed the intervention and were included in the statistical evaluation (Fig. 1; Table 3); see the "Materials and methods" section for a comprehensive description regarding selection of individuals and exclusion criteria. The microbiota composition with respect to Prevotella and Bacteroides of the 59 subjects that were not included in the short-term intervention, are displayed in Fig. 1.

\section{Effects of BKB vs WWB interventions on metabolic markers in subgroups varying in baseline gut microbiota composition}

To investigate if abundance of Prevotella and Prevotella/Bacteroides ratios can predict the metabolic response to $\mathrm{BKB}$, we analyzed the metabolic responses in the subjects from the different microbiota subgroups (HP, HPB and LP).

\section{Glucose and insulin}

A main effect of treatment was observed on incremental postprandial b-glucose responses (iAUC 0-150 $\mathrm{min}$ ) to the standardized breakfast $(2 \times 3$ factorial design, $P<0.01)$, indicating that the $\mathrm{BKB}$ intervention significantly lowered the b-glucose responses, compared to the WWB, in all three subgroups, i.e., independently of the baseline gut microbiota (Fig. 2). No main effects of intervention were detected on insulin responses to the standardized breakfast. Instead, baseline gut microbiota tended to impact the incremental postprandial s-insulin responses (iAUC 0-150 $\mathrm{min}$ ) post the standardized breakfast $(2 \times 3$ factorial design, $P=0.070)$, independently of intervention (Fig. 2).

The HPB group was excluded to facilitate the elucidation of differences between the "extremes" with respect to

Table 2 Age and BMI of the subjects in the three different microbiota subgroups as well as in the total group

\begin{tabular}{llll}
\hline Microbiota group & Gender $(n$ subjects $)$ & Age (years) & BMI $\left(\mathrm{kg} / \mathrm{m}^{2}\right)$ \\
\hline HP $(n=12)$ & $3 \mathrm{M} / 9 \mathrm{~W}$ & $60.9 \pm 7.1$ & $24.1 \pm 3.3$ \\
LP $(n=13)$ & $2 \mathrm{M} / 11 \mathrm{~W}$ & $65.8 \pm 5.1$ & $24.2 \pm 2.7$ \\
HPB $(n=8)$ & $4 \mathrm{M} / 4 \mathrm{~W}$ & $65.8 \pm 2.9$ & $23.6 \pm 3.9$ \\
Total $(n=33)$ & $9 \mathrm{M} / 24 \mathrm{~W}$ & $64.0 \pm 5.8$ & $24.0 \pm 3.2$ \\
\hline
\end{tabular}

Data including age and BMI are presented as means \pm SD

$P$ Prevotella, $B$ Bacteroides, $M$ men, $W$ women 

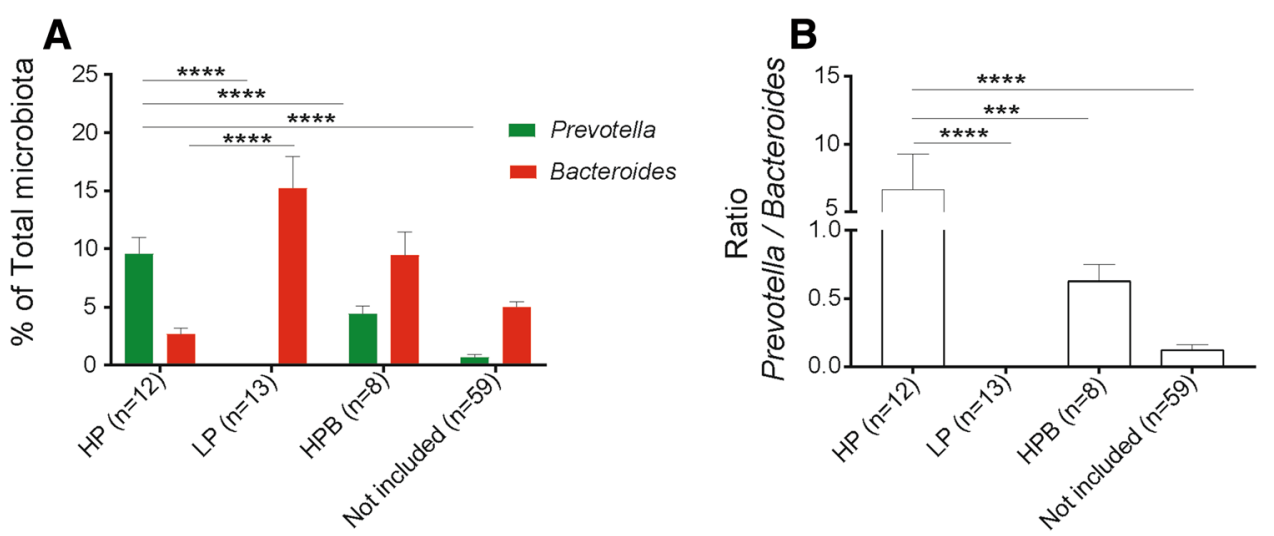

Fig. 1 Baseline levels of fecal bacteria and stratification of individuals. a Fecal levels of Prevotella and Bacteroides (determined by quantitative PCR and expressed as fraction of the total microbiota) in participants included and not included in the short-term barley prebiotic intervention. b Ratio of Prevotella vs Bacteroides in participants included and not included in the short-term barley prebiotic interven- tion. Included HP (High Prevotella), LP (Low Prevotella) and HPB (High Prevotella and Bacteroides). ANOVA followed by post hoc analysis-Tukey's pairwise multiple comparison method was used to compare changes in the levels of Prevotella and Bacteroides and the ratio Prevotella/Bacteroides across the four groups. Data are mean \pm SEM. $* * * P<0.001, * * * * P<0.0001$
Table 3 Abundance of Prevotella and Bacteroides, and Prevotella/Bacteroides ratio of the subjects in the three different microbiota subgroups

\begin{tabular}{llll}
\hline Microbiota group & Prevotella, 16S copies & Bacteroides, 16S copies & P/B ratio \\
\hline HP $(n=12)$ & $1.32 \times 10^{8} \pm 1.05 \times 10^{8}$ & $3.90 \times 10^{7} \pm 3.29 \times 10^{7}$ & $6.67 \pm 8.93$ \\
LP $(n=13)$ & $1.12 \times 10^{5} \pm 1.13 \times 10^{5}$ & $1.43 \times 10^{8} \pm 8.77 \times 10^{7}$ & 0 \\
HPB $(n=8)$ & $5.27 \times 10^{7} \pm 4.56 \times 10^{7}$ & $1.00 \times 10^{8} \pm 7.76 \times 10^{7}$ & $0.63 \pm 0.34$ \\
\hline
\end{tabular}

Data are presented as means $\pm \mathrm{SD}$

$P / B$ ratio Prevotella/Bacteroides ratio the abundance of Prevotella (HP vs LP). When comparing s-insulin responses (iAUC 0-150 min) in these subgroups, we observed that the HP group had an overall lower insulin response, compared to the LP group $(2 \times 2$ factorial design, $P<0.05$ ) (Fig. 2). No main effects of treatment or baseline microbiota were observed on fasting b-glucose and s-insulin concentrations (both ANOVA $2 \times 3$ and $2 \times 2$ factorial designs, $P>0.05$ ) (Table 4 ).

When investigating the treatment effects on b-glucoseand s-insulin concentrations in each subgroup separately (using one-way ANOVA), it was observed that 3-day BKB intervention, in comparison with the WWB intervention, increased the fasting b-glucose concentrations in the LP group $(P<0.05)$, whereas no such effects were seen in the other two subgroups $(P>0.05)$ (Table 4$)$. The incremental postprandial s-insulin responses after the standardized breakfast (AUC 0-150 min and iAUC 0-150 min) were decreased after BKB intervention compared with WWB intervention in two subgroups: HP and LP, $(P<0.05$, Table 4; Fig. 2). In addition, in the HP group, the BKB intervention showed a trend $(-6 \%, P=0.067)$ towards lowering the postprandial b-glucose response (AUC 0-150 min), see Table 4 and Fig. 2.

\section{Gut hormones}

No significant main effects of treatment or baseline microbiota composition were observed at fasting or with respect to mean concentrations of the gut hormones PYY, GLP-1 or GLP- 2 using the ANOVA $2 \times 3$ (all 3 subgroups) or $2 \times 2$ (without HPB) factorial designs (Fig. 3 and data are displayed in Online resource 1). However, evaluation of treatment effects on gut hormone concentrations in each subgroup separately (using one-way ANOVA) displayed that the group HPB acquired significantly increased mean GLP-1 and GLP-2 concentrations (0-180 min) with 12 and $6 \%$ respectively, after BKB intake compared to after WWB $(P=0.018$ and $P=0.034)$ (Fig. 3 and Online resource 1). In the HP group, there was a trend towards increased fasting concentration of p-PYY $(11 \%, P=0.050)$ and mean 
Fig. 2 Incremental b-glucose (a) and s-insulin (b) responses post the standardized breakfast after BKB or WWB interventions, respectively, in subgroups varying in baseline gut microbiota composition. One-way ANOVA was used to compare the metabolic responses depending on intervention in each subgroup. The percentage corresponds to differences in concentration of test variables after BKB intervention compared to WWB intervention. $B K B$ barley kernel bread, $W W B$ white wheat bread, $H P$ high Prevotella, LP low Prevotella and $H P B$ high Prevotella and Bacteroides. Data are mean \pm SEM. $* P<0.05$
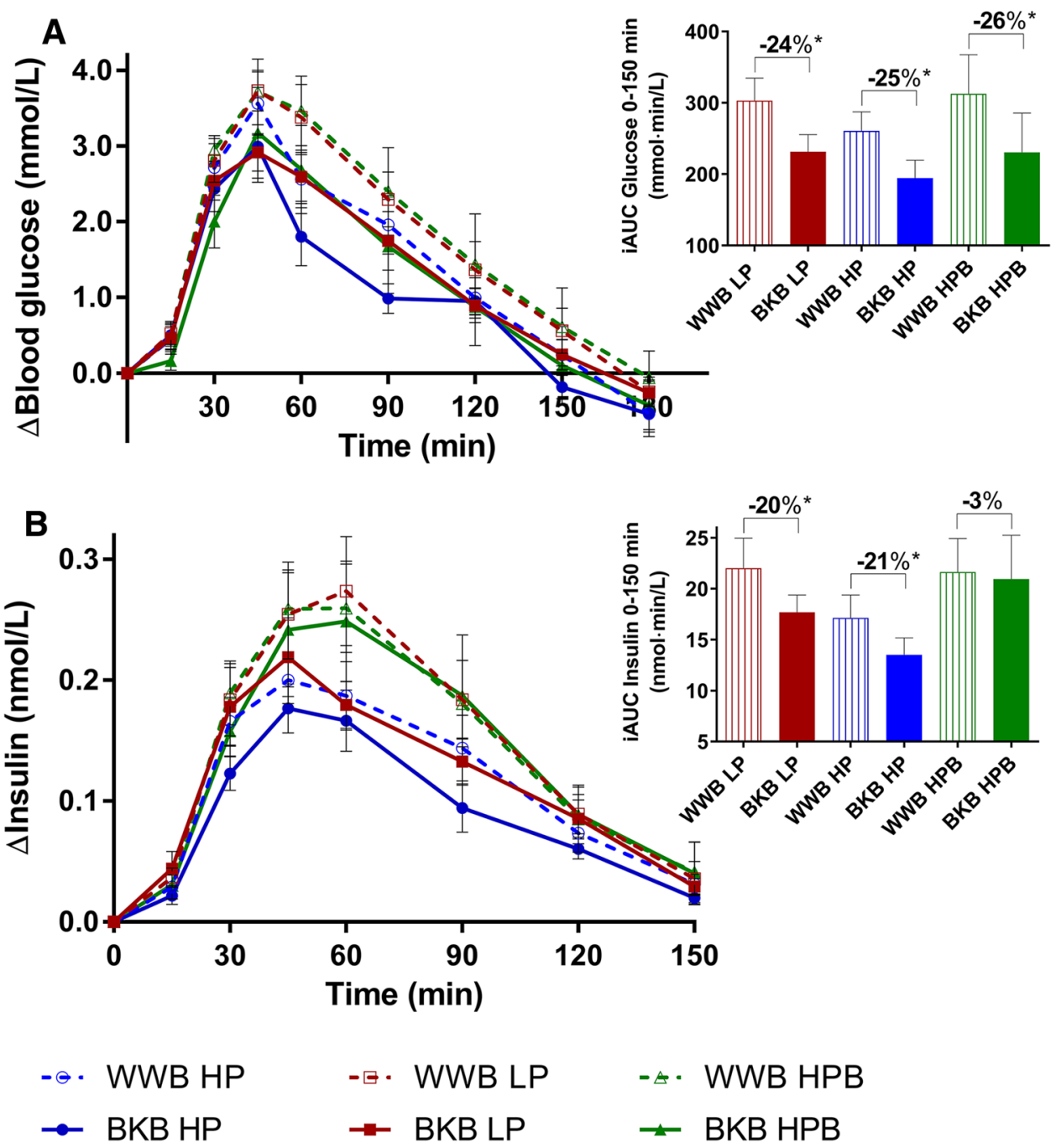

concentrations of p-PYY $(0-180 \mathrm{~min},+9 \%, P=0.060)$ in the morning the day after the BKB intervention, compared to after WWB intervention (Fig. 3 and Online resource 1).

\section{Breath hydrogen}

Breath $\mathrm{H}_{2}$ levels at both the fasting and mean (0-180 min) displayed a main effect of treatment $(2 \times 3$ factorial design, $P<0.001)$ and of baseline gut microbiota composition $(2 \times 3$ factorial design, $P<0.05)$ (data are presented in Online resource 1). The post hoc analysis (Tukey's method) showed that the BKB intervention significantly increased the breath $\mathrm{H}_{2}$ excretion in all subgroups, compared to WWB intervention $(P<0.001)$, and revealed that fasting $\mathrm{H}_{2}$ levels were higher in the LP group compared with the HP group $(P=0.017)($ Online resource 1$)$.

\section{Markers of inflammation}

No significant main effects depending on treatment or baseline gut microbiota composition were observed regarding the concentrations of p-IL- 6 and p-CRP in the ANOVA $2 \times 3$ factorial design (Fig. 4 and data are displayed in Online resource 2). However, comparing results in the HP and LP subgroups only $(2 \times 2$ factorial design), a main effect of baseline gut microbiota composition was detected on concentrations of p-IL-6 (mean 0-180 min, $P=0.045$ ), revealing lower IL-6 concentrations in the HP group (Fig. 4 and Online resource 2). In addition, the comparison of the CRP fasting concentrations in the subgroups HP and LP displayed a trend towards a main effect of baseline gut microbiota composition $(2 \times 2$ factorial design, $P=0.084)$, indicating a trend towards lower CRP concentrations in the HP group (Fig. 4 and Online resource 2). No significant differences in concentrations of p-IL- 6 or p-CRP were seen within the separate microbiota subgroups depending on treatment (oneway ANOVA, $P>0.05$ ) (Fig. 4 and Online resource 2). 
Table 4 Fasting and postprandial measures of b-glucose, s-insulin and insulin sensitivity post the standardized breakfast after BKB or WWB, respectively, in subgroups varying in baseline gut microbiota composition

\begin{tabular}{|c|c|c|c|c|c|}
\hline & \multicolumn{2}{|l|}{ WWB } & \multicolumn{3}{|l|}{ BKB } \\
\hline & Mean & SEM & Mean & SEM & $\% \Delta^{\mathrm{a}}$ \\
\hline \multicolumn{6}{|l|}{ Glucose } \\
\hline \multicolumn{6}{|c|}{ b-Glucose, fasting (mmol/L) } \\
\hline $\mathrm{HP}(n=12)$ & 5.6 & 0.1 & 5.6 & 0.1 & 1 \\
\hline $\mathrm{LP}(n=13)$ & 5.3 & 0.2 & 5.5 & 0.2 & $6^{*}$ \\
\hline HPB $(n=8)$ & 5.5 & 0.2 & 5.7 & 0.2 & 5 \\
\hline \multicolumn{6}{|c|}{ b-Glucose, AUC 0-150 min (mmol-min/L) } \\
\hline $\mathrm{HP}(n=12)$ & 1090 & 38 & 1030 & 30 & $-6^{\dagger}$ \\
\hline $\mathrm{LP}(n=13)$ & 1088 & 39 & 1062 & 39 & -2 \\
\hline $\mathrm{HPB}(n=8)$ & 1127 & 67 & 1077 & 59 & -4 \\
\hline \multicolumn{6}{|l|}{ Insulin } \\
\hline \multicolumn{6}{|c|}{ s-Insulin, fasting (nmol/L) } \\
\hline $\mathrm{HP}(n=12)$ & 0.030 & 0.004 & 0.032 & 0.005 & 8 \\
\hline $\mathrm{LP}(n=13)$ & 0.029 & 0.003 & 0.027 & 0.003 & -6 \\
\hline $\operatorname{HPB}(n=8)$ & 0.029 & 0.005 & 0.033 & 0.006 & 13 \\
\hline \multicolumn{6}{|c|}{ s-Insulin, AUC 0-150 min (nmol min/L) } \\
\hline $\mathrm{HP}(n=12)$ & 21.6 & 2.7 & 18.3 & 2.2 & $-15^{*}$ \\
\hline $\mathrm{LP}(n=13)$ & 26.4 & 3.3 & 21.7 & 2.0 & $-18 *$ \\
\hline $\operatorname{HPB}(n=8)$ & 26.0 & 4.0 & 25.9 & 5.0 & 0 \\
\hline \multicolumn{6}{|l|}{ Insulin sensitivity } \\
\hline \multicolumn{6}{|l|}{ HOMA-IR } \\
\hline $\mathrm{HP}(n=12)$ & 1.23 & 0.17 & 1.34 & 0.22 & 8 \\
\hline $\mathrm{LP}(n=13)$ & 1.12 & 0.14 & 1.14 & 0.13 & 2 \\
\hline HPB $(n=8)$ & 1.22 & 0.26 & 1.42 & 0.26 & 16 \\
\hline \multicolumn{6}{|l|}{ ISI $_{\text {composite }}$} \\
\hline $\mathrm{HP}(n=12)$ & 9.44 & 1.14 & 11.05 & 1.96 & 17 \\
\hline $\mathrm{LP}(n=13)$ & 8.96 & 0.98 & 9.59 & 1.06 & 7 \\
\hline$(n=8)$ & 10.2 & 2.43 & 8.97 & 1.68 & -12 \\
\hline
\end{tabular}

Data are presented as mean \pm SEM

$B K B$ barley kernel bread, $W W B$, white wheat bread

*Different from WWB $P<0.05,{ }^{\dagger} P=0.067$ (One-way ANOVA)

${ }^{a}$ The percentage change is calculated as the difference from the WWB

\section{Free fatty acids}

No significant main effects depending on treatment or baseline gut microbiota composition were observed regarding the concentrations of s-FFA in the ANOVA $2 \times 3$ or $2 \times 2$ factorial design (data are displayed in Online resource 2). Neither were there any significant differences in concentrations of FFA within the separate microbiota subgroups depending on treatment (one-way ANOVA, $P>0.05$ ).

\section{Subjective appetite ratings}

The postprandial appetite ratings 'hunger' and 'desire to eat' (AUC 0-180 min) displayed main effects of baseline gut microbiota composition $(2 \times 3$ factorial, hunger, $P<0.05$; desire to eat, $P<0.01$ ) (Fig. 5 and data are presented in Online resource 3). The post hoc analysis (Tukey's method) showed a significantly higher sensations of hunger and desire to eat in the LP group compared to both the HP group (hunger, $P<0.05$; desire to eat, $P<0.01$ ) and the HPB group (both variables, $P<0.05$ ) (Fig. 5 and Online resource 3 ). No significant main effects were seen on subjective appetite ratings depending on treatment. However, investigations of effects of treatment in each subgroup separately, analyzed using one-way ANOVA, revealed significant effects of treatment on appetite ratings in the LP group. Consequently, after the BKB intervention, the subgroup LP significantly decreased sensations of hunger at fasting $(-28 \%, P<0.05)$ and increased fasting satiety $(+92 \%, P<0.01)$ (Online resource 3 ), and showed a trend to decrease postprandial desire to eat (AUC $0-180 \mathrm{~min},-15 \%, P=0.082$ ) (Fig. 5 and Online resource 3 ), compared to the ratings after WWB intake.

\section{Discussion}

The purpose of this study was to investigate if the abundance of gut Prevotella and the ratio of Prevotella/Bacteroides at baseline may predict the effects of barley kernel-based food on cardiometabolic risk variables in a time perspective of, e.g., 11-13.5 $\mathrm{h}$ after intake. Therefore, subjects were stratified into different subgroups (HP, LP and HPB) depending on the gut microbiota composition. We evaluated both the effects of the short-term 3-day interventions with BKB vs WWB and effects of microbiota subgroups on cardiometabolic test variables. Effects of the interventions were also evaluated within each microbiota subgroup. The current design, 3-day long intervention with $\mathrm{BKB}$, was based on previous findings where 3 days of intake of BKB was sufficient to observe acute effects of gut microbiota and glucose metabolism [10].

All subgroups responded to the BKB intervention with improved glucose tolerance $11 \mathrm{~h}$ after intake as compared with the WWB intervention, thus, the results were not dependent on the subgroup stratification. Instead, we identified significant differences in cardiometabolic test markers between the microbiota subgroups independently of the intervention. Furthermore, the beneficial response to BKB can be driven by a substrate depending expansion of Prevotella, regardless of the baseline levels gut Prevotella, which can mediate the improved glucose metabolism, in agreement to our previous findings [10]. 
Fig. 3 Mean concentrations of p-GLP-1 (a), p-GLP-2 (b) and p-PYY (c) after BKB or WWB intervention, respectively, in subgroups varying in gut microbiota composition. One-way ANOVA was used to compare the metabolic responses depending on intervention in each subgroup. The percentage corresponds to differences in concentration of test variables after BKB intervention compared to WWB intervention. $G L P$ glucagon-like peptide, $P Y Y$ peptide YY, $B K B$ barley kernel bread, $W W B$ white wheat bread, $H P$ high Prevotella, $L P$ low Prevotella and $H P B$ high Prevotella and Bacteroides. Data are mean $\pm \mathrm{SEM}$. ${ }^{*} P<0.05,{ }^{\#} P=0.06$
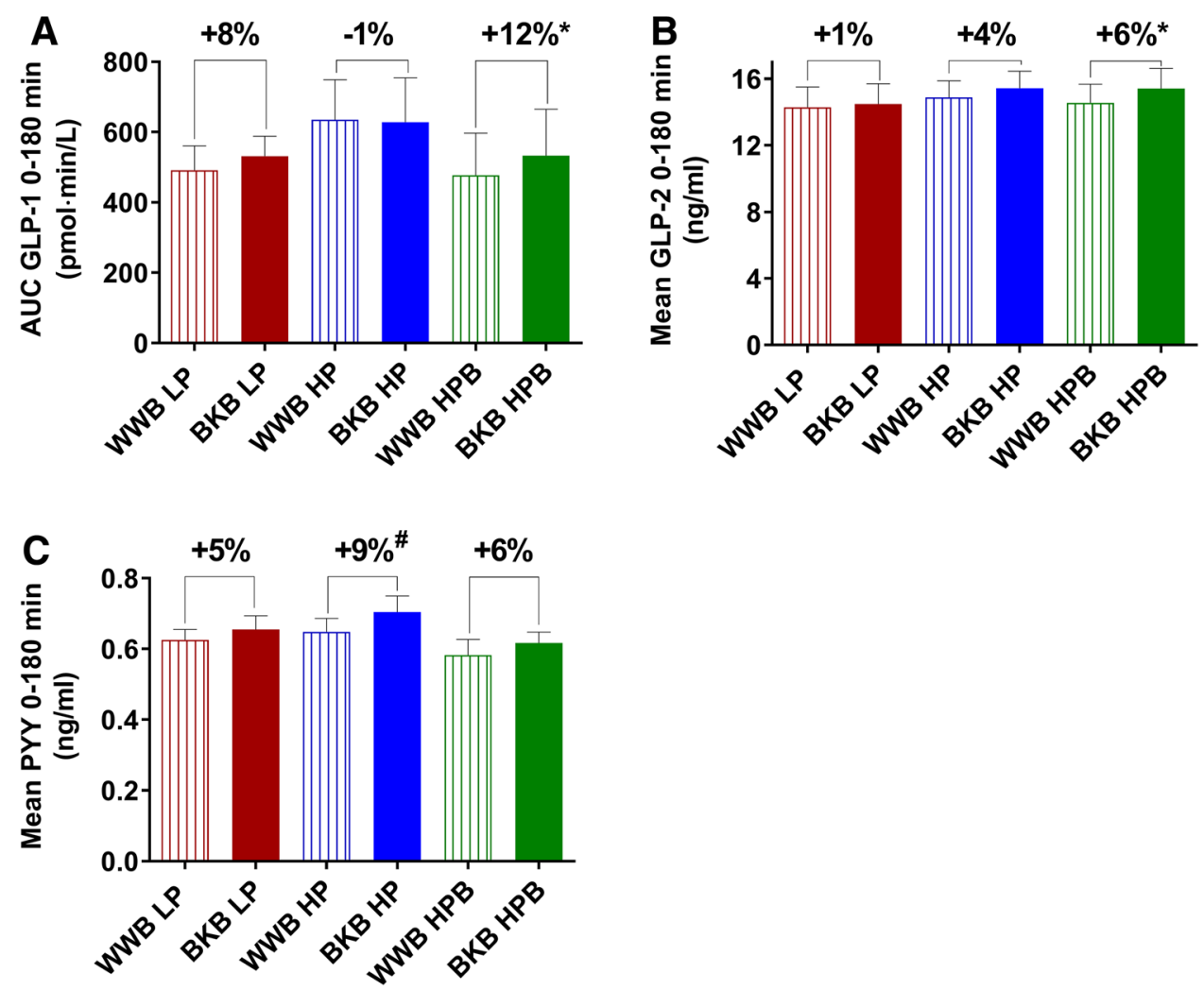

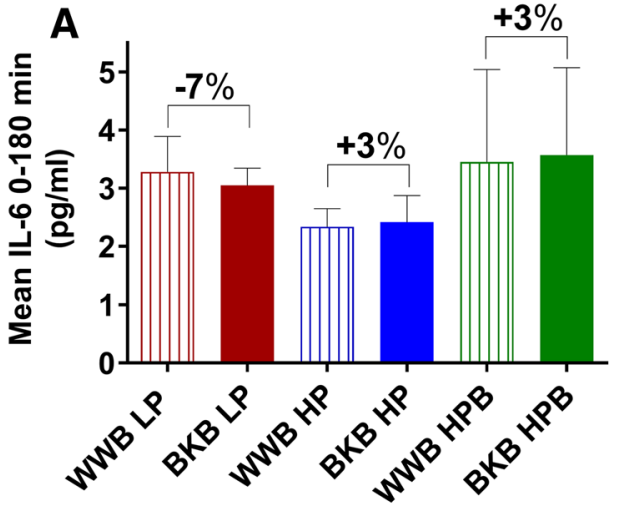

Fig. 4 Plasma concentrations of IL-6 (a) after the standardized breakfast and fasting values of CRP (b) after BKB or WWB intervention, respectively, in subgroups varying in gut microbiota composition. One-way ANOVA was used to compare the metabolic responses depending on intervention in each subgroup. The percentage cor-

Colonic fermentation of DF endogenous to barley kernels has been related to improved cardiometabolic risk markers in healthy subjects [6,7]. However, high variability between subjects in the metabolic responses to food, e.g., postprandial glycemic responses, or SCFA production (in particular butyrate), has previously been observed, which may be related to gut microbiota composition [11, 12, 22]. In this context, we have previously shown that improved glucose

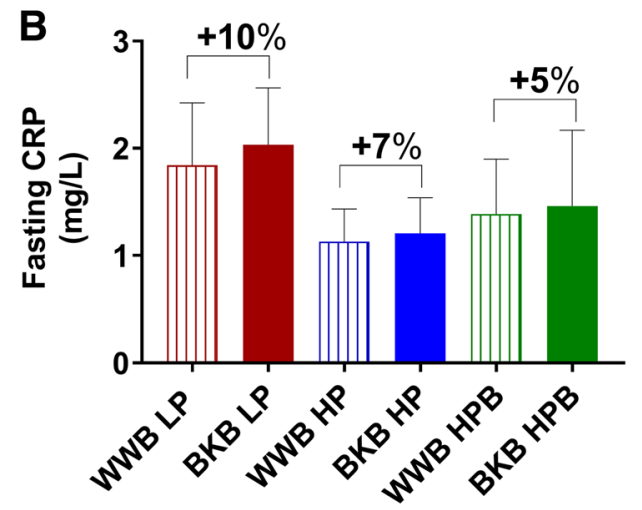

responds to differences in concentration of test variables after $\mathrm{BKB}$ intervention compared to WWB intervention. $I L$ interleukin, $C R P$ c-reactive protein, $B K B$ barley kernel bread, $W W B$ white wheat bread, HP high Prevotella, LP low Prevotella and HPB high Prevotella and Bacteroides. Data are mean \pm SEM

tolerance after a short-term intervention with BKB was related to an enrichment of gut Prevotella, where $P$. copri was the most prevalent [10]. In addition, subjects responding to BKB with an improved glucose tolerance tended to have an elevated abundance of Prevotella also at baseline, prior to the BKB intervention. Prevotella can ferment a wide spectrum of complex carbohydrates, such as arabinoxylans and $\beta$-glucans, i.e., DF abundant in barley [23]. Populations 
Fig. 5 Subjective appetite ratings [subjective hunger (a), desire to eat (b) and satiety (c)] post standardized breakfast after BKB or WWB intervention, respectively, in subgroups varying in gut microbiota composition. One-way ANOVA was used to compare the metabolic responses depending on intervention in each subgroup. The percentage corresponds to differences in concentration of test variables after BKB intervention compared to WWB intervention. $B K B$ barley kernel bread, $W W B$ white wheat bread, $H P$ high Prevotella, $L P$ low Prevotella and HPB high Prevotella and Bacteroides. Data are mean \pm SEM. ${ }^{*} \mathrm{P}=0.065$
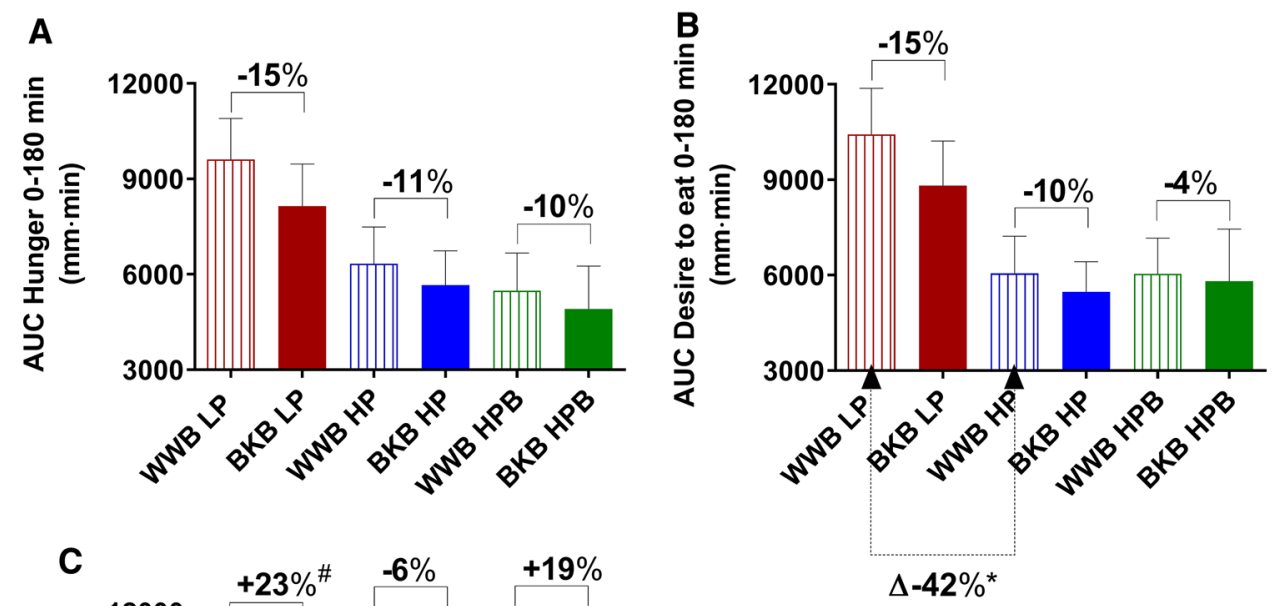

$\Delta-42 \%$ *

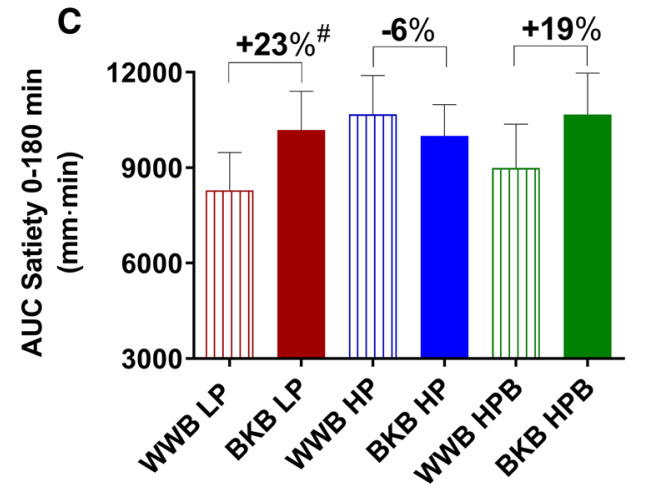

living in rural areas ingesting diets rich in DF have higher abundance of Prevotella compared to those on a Western diet [24]. Accordingly, Prevotella has been associated with high intake of carbohydrates/dietary fiber [25].

With respect to the metabolic effects of barley kernelbased products, the results in the current study were in accordance with previous results showing beneficial effects on markers of glucose regulation, gut hormones, subjective appetite variables, and increased breath $\mathrm{H}_{2}$ excretion $[6,7$, $26,27]$. Consequently, compared to WWB, the BKB intervention resulted in improvements with respect to glucose tolerance in all subgroups, and insulin responses decreased in the subgroups HP and LP. Furthermore, gut hormones (GLP-1 and GLP-2) increased after BKB in the HPB group in the postprandial phase, whereas there was a trend $(P=0.05)$ towards increased PYY fasting concentrations in the HP group. In addition, the BKB intervention resulted in decreased sensation of hunger and increased satiety after BKB intervention in the LP group. Breath $\mathrm{H}_{2}$ excretion was increased in all subgroups after the BKB intervention.

Interestingly, the main effects of baseline gut microbiota composition (HP, LP or HPB) were on appetite variables (hunger and desire to eat) and breath $\mathrm{H}_{2}$, independent of the intervention. Thus, although the LP group improved appetite ratings after the BKB intervention, the post hoc analysis revealed that both subgroups with higher gut Prevotella abundance, i.e., the subgroups HP and HPB, in general displayed improved appetite sensations compared with the LP group with respect to less hunger and less desire to eat, independently of intervention. In addition, it was shown that the HP group had significantly lower breath $\mathrm{H}_{2}$ concentrations at fasting, compared to the LP group. These findings further suggest differences in the gut microbiota fermentative activities between these two groups. Furthermore, the differences in insulin responses to the standardized breakfast between the microbiota subgroups that differ the most in composition, i.e., the HP and LP groups, revealed a significant effect of baseline gut microbiota composition, demonstrating that a high abundance of Prevotella and high Prevotella/Bacteroides ratio at baseline may be advantageous with respect to postprandial glucose homeostasis, compared to a low baseline Prevotella and low Prevotella/Bacteroides ratio.

Upon designing the study, we included 99 subjects with the intention to identify 20 subjects to be included in the HP group and 20 subjects in the LP group. However, following the initial microbial screening, we could not identify 20 subjects with high or low Prevotella, which limits the study design. Surprisingly, we identified subjects with relatively high levels of both Prevotella and Bacteroides (the HPB group), which is interesting as taxa belonging to these genera usually are believed to compete for the same niche [28]. 
In the present study, we focused on the potential changes in cardiometabolic test variables after BKB compared to WWB in healthy subjects after a short-term intervention depending on specific bacteria ratios. For future work, it would be interesting to design a longer term study and it could also be of interest to focus on prediabetic cohort and/ or different ages. Furthermore, the screening criteria for healthy subjects would also benefit from including $\mathrm{HbA} 1 \mathrm{c}$ measurements to obtain further indication of the glycemic status.

In summary, the BKB intervention resulted in improved glucose tolerance after the forthcoming standardized breakfast in all microbiota subgroups, suggesting overall prebiotic and anti-diabetic potentials of barley DF independently of Prevotella/Bacteroides ratio at baseline. There were no differences between the microbiota subgroups in response to $\mathrm{BKB}$, in comparison to WWB, with respect to the tested variables. Thus, our results do not support the hypothesis that the Prevotella/Bacteroides ratio at baseline can be used to stratify metabolic responders and non-responders to the prebiotic mixture present in BKB. However, these findings do not exclude that the beneficial effects of DF are mediated by Prevotella-dependent fermentation. In addition, based on reduced insulin responses, reduced inflammatory markers and improved subjective appetite ratings in the HP group compared to the LP group (independent of intervention), the results of the current study suggest that higher Prevotella/Bacteroides ratio may be advantageous with respect to cardiometabolic regulation, and may play a preventive role in the development of obesity, T2D and CVD.

Acknowledgements We thank L Persson at department of Food Technology, Engineering and Nutrition, Lund University, Sweden, for her skillful technical assistance. This study was supported by Antidiabetic Food Center-a VINNOVA Centre of Excellence in Research and Innovation at Lund University, the Swedish Research Council, and the NovoNordisk Foundation. FB is recipient of an ERC Consolidator Grant (European Research Council, Consolidator grant 615362-METABASE), and FB is awarded Torsten Söderberg Professorship in Medicine.

Author contributions JS, PKD, IB, FB and AN conceived and designed the project. JS, PKD and AN performed experiments. JS, PKD, IB, FB and AN analyzed and interpreted the data. JS, PKD, IB, FB and AN wrote the paper.

\section{Compliance with ethical standards}

Conflict of interest AN and IB are founders and shareholders of ProPrev AB.

Ethical approval Dnr 2015/58.

Open Access This article is distributed under the terms of the Creative Commons Attribution 4.0 International License (http://creat ivecommons.org/licenses/by/4.0/), which permits unrestricted use, distribution, and reproduction in any medium, provided you give appropriate credit to the original author(s) and the source, provide a link to the Creative Commons license, and indicate if changes were made.

\section{References}

1. Grundy SM (2016) Metabolic syndrome update. Trends Cardiovasc Med 26(4):364-373

2. Singh RK et al (2017) Influence of diet on the gut microbiome and implications for human health. Jo Transl Med 15(1):73

3. Conlon MA, Bird AR (2015) The impact of diet and lifestyle on gut microbiota and human health. Nutrients 7(1):17-44

4. Ye EQ et al (2012) Greater whole-grain intake is associated with lower risk of type 2 diabetes, cardiovascular disease, and weight gain. J Nutr 142(7):1304-1313

5. Grooms KN et al (2013) Dietary fiber intake and cardiometabolic risks among US adults, NHANES 1999-2010. Am J Med 126(12): 1059e1-e4-1067e1-e4

6. Nilsson AC, Johansson-Boll EV, Bjorck IM (2015) Increased gut hormones and insulin sensitivity index following a 3-d intervention with a barley kernel-based product: a randomised crossover study in healthy middle-aged subjects. Br J Nutr 114:1-9

7. Priebe MG et al (2010) Factors related to colonic fermentation of nondigestible carbohydrates of a previous evening meal increase tissue glucose uptake and moderate glucose-associated inflammation. Am J Clin Nutr 91(1):90-97

8. Ibrugger $\mathrm{S}$ et al (2014) Second meal effect on appetite and fermentation of wholegrain rye foods. Appetite 80:248-256

9. Sandberg JC, Bjorck IM, Nilsson AC (2016) Rye-based evening meals favorably affected glucose regulation and appetite variables at the following breakfast; a randomized controlled study in healthy subjects. PLoS One 11(3):e0151985

10. Kovatcheva-Datchary P et al (2015) Dietary fiber-induced improvement in glucose metabolism is associated with increased abundance of prevotella. Cell Metab 22(6):971-982

11. Zeevi D et al (2015) Personalized nutrition by prediction of glycemic responses. Cell 163(5):1079-1094

12. Korem $\mathrm{T}$ et al (2017) Bread affects clinical parameters and induces gut microbiome-associated personal glycemic responses. Cell Metab 25(6):1243-1253.e5

13. Salonen A et al (2010) Comparative analysis of fecal DNA extraction methods with phylogenetic microarray: effective recovery of bacterial and archaeal DNA using mechanical cell lysis. J Microbiol Methods 81(2):127-134

14. Walker AW et al (2011) Dominant and diet-responsive groups of bacteria within the human colonic microbiota. ISME J $5(2): 220-230$

15. Matsuki T et al (2002) Development of $16 \mathrm{~S}$ rRNA-gene-targeted group-specific primers for the detection and identification of predominant bacteria in human feces. Appl Environ Microbiol 68(11):5445-5451

16. World Health Organization (2006) Definition and diagnosis of diabetes mellitus and intermediate hyperglycemia: report of a WHO/IDF Consultation

17. Björck IME, Siljeström MA (1992) In-vivo and in-vitro digestibility of starch in autoclaved pea and potato products. J Sci Food Agric 58(4):541-553

18. Akerberg AK et al (1998) An in vitro method, based on chewing, to predict resistant starch content in foods allows parallel determination of potentially available starch and dietary fiber. J Nutr 128(3):651-660

19. Asp NG et al (1983) Rapid enzymatic assay of insoluble and soluble dietary fiber. J Agric Food Chem 31(3):476-482 
20. Matthews DR et al (1985) Homeostasis model assessment: insulin resistance and beta-cell function from fasting plasma glucose and insulin concentrations in man. Diabetologia 28(7):412-419

21. Matsuda M, DeFronzo RA (1999) Insulin sensitivity indices obtained from oral glucose tolerance testing: comparison with the euglycemic insulin clamp. Diabetes Care 22(9):1462-1470

22. Venkataraman A et al (2016) Variable responses of human microbiomes to dietary supplementation with resistant starch. Microbiome 4(1):33

23. Gorham JB et al (2016) Addition of arabinoxylan and mixed linkage glucans in porcine diets affects the large intestinal bacterial populations. Eur J Nutr 56:2193-2206

24. De Filippo C et al (2010) Impact of diet in shaping gut microbiota revealed by a comparative study in children from Europe and rural Africa. Proc Natl Acad Sci USA 107(33):14691-14696
25. Wu GD et al (2011) Linking long-term dietary patterns with gut microbial enterotypes. Science 334(6052):105-108

26. Johansson EV et al (2013) Effects of indigestible carbohydrates in barley on glucose metabolism, appetite and voluntary food intake over $16 \mathrm{~h}$ in healthy adults. Nutr J 12:46

27. Nilsson AC et al (2008) Including indigestible carbohydrates in the evening meal of healthy subjects improves glucose tolerance, lowers inflammatory markers, and increases satiety after a subsequent standardized breakfast. J Nutr 138(4):732-739

28. Arumugam $\mathrm{M}$ et al (2011) Enterotypes of the human gut microbiome. Nature 473(7346): 174-180 